\title{
As if one pain problem was not enough: prevalence and patterns of coexisting chronic pain conditions and their impact on treatment outcomes
}

This article was published in the following Dove Press journal: Journal of Pain Research

\author{
M Gabrielle Pagé ${ }^{1,2}$ \\ Maude Fortier' \\ Mark A Ware ${ }^{3-5}$ \\ Manon Choinière ${ }^{1,6}$ \\ 'Centre de recherche du Centre \\ hospitalier de l'Université de \\ Montréal (CRCHUM), ${ }^{2}$ Department \\ of Biomedical Sciences, Faculty of \\ Medicine, Université de Montréal, \\ ${ }^{3}$ Department of Family Medicine, \\ ${ }^{4}$ Department of Anesthesia, Faculty of \\ Medicine, ${ }^{5}$ Alan Edwards Centre for \\ Research on Pain, McGill University, \\ ${ }^{6}$ Department of Anesthesiology, \\ Faculty of Medicine, Université de \\ Montréal, Montreal, QC, Canada
}

Introduction: The presence of multiple coexisting chronic pain (CP) conditions (eg, low-back pain and migraines) within patients has received little attention in literature. The goals of this observational longitudinal study were to determine the prevalence of coexisting $\mathrm{CP}$ conditions, identify the most frequent ones and patterns of coexistence, investigate the relationships among patients' biopsychosocial characteristics and number of CP conditions, and determine the impact of coexisting CP conditions on treatment response.

Patients and methods: A total of 3,966 patients attending multidisciplinary pain-treatment centers who were enrolled in the Quebec Pain Registry were included. Patients completed selfreport and nurse-administered questionnaires before their first visit and 6 months later. Results were analyzed using descriptive statistics, factor and cluster analyses, negative binomials with log-link generalized linear models, and linear mixed-effect models.

Results: A third of patients reported coexisting CP conditions. No specific patterns of comorbidities emerged. The presence of coexisting CP conditions was associated with longer pain duration, older age, being female, and poorer quality of life. The presence of more than one CP condition did not have a clinically significant impact on treatment responses.

Discussion: The novelty of the study results relate to the heterogeneity that was found in the patterns of coexistence of CP conditions and the fact that having multiple CP conditions did not clinically impact treatment response. These results highlight the need for future research that examines causes of coexistence among CP conditions across the spectrum of $\mathrm{CP}$, as opposed to focusing on specific conditions, and to examine whether multiple $\mathrm{CP}$ conditions impact on additional domains, such as treatment satisfaction. These results highlight the importance of studying the pathophysiological mechanisms underlying the development of coexisting CP conditions, in order eventually to prevent/minimize their occurrence and/or develop optimal treatment and management approaches.

Keywords: chronic pain, coexisting pain conditions, comorbidities, Quebec Pain Registry, cluster analysis

\section{Introduction}

Chronic pain (CP), defined as pain persisting for longer than normal/expected tissuehealing time (3-6 months) or associated with a progressive nonmalignant disease, ${ }^{1,2}$ is experienced by up to $20 \%$ of the population. ${ }^{3-5}$ Importantly, CP is now recognized as a disease entity and not merely a symptom of underlying diseases. ${ }^{6,7}$

Comorbidity between $\mathrm{CP}$ and other conditions, such as psychiatric conditions, has been well documented. ${ }^{8}$ The presence of comorbid conditions is important, as it has
Correspondence: Manon Choinière Centre de recherche du Centre hospitalier de l'Université de Montréal (CRCHUM), Tour Saint-Antoine, bureau SOI-I26, 850 Rue Saint-Denis, Montreal, QC H2X 0A9 Canada

Tel + I 5 I48908000 ext I4082

Email manon.choiniere@umontreal.ca 
been shown to increase health-care utilization and health-care costs and diminish health-related quality of life. ${ }^{9} 10$ Specific to pain comorbidities, patients with multiple pain sites or pain syndromes also report higher levels of anxiety and depressive symptoms ${ }^{11,12}$ and make greater use of more health-care resources. ${ }^{13}$ However, research in the field of coexisting CP conditions remains scarce. ${ }^{10,14}$

Some studies have documented the prevalence of multiple pain sites. For example, in Picavet and Schouten's survey, close to $20 \%$ of patients with CP reported pain symptoms in more than one body area. ${ }^{15}$ This does not suggest coexisting pain conditions, however, as some individual pain conditions (eg, fibromyalgia) are by definition present in multiple body sites. There is a lack of research documenting coexistence of comorbid pain conditions; most of the literature typically focuses on the prevalence of other pain conditions among patients presenting with an indexed pain condition. ${ }^{10}$ For example, research has shown that patients with low-back pain often experience coexisting musculoskeletal pain or neuropathic pain problems ${ }^{11,16}$ and patients with irritable bowel syndrome are more likely also to suffer from fibromyalgia, back pain, or migraines. ${ }^{13}$

Studies examining patterns of coexisting pain conditions are lacking, so the underlying mechanisms are poorly understood. Some specific mechanisms, such as a generalized vulnerability to develop CP or shared risk factors, have been proposed. ${ }^{14}$ Risk factors for $\mathrm{CP}$, such as older age and being female, ${ }^{5,17}$ however, do not have a uniform impact on pain prevalence across diagnoses. For example, reviews on sex differences in pain concluded that this relationship is complex and not uniform across conditions. ${ }^{18-20}$ Similarly, the risk of developing some CP conditions is higher in younger age (eg, chronic postsurgical pain), ${ }^{21,22}$ whereas risk increases over time for other conditions (eg, fibromyalgia). ${ }^{23}$

It has been documented that the presence of comorbid physical or mental health conditions or symptoms has an important clinical impact, notably in terms of treatment responses. $^{24,25}$ For example, a study examining a specific cluster of pain-related symptoms (sleep disturbances, pain, anxiety, depression, and low energy/fatigue) showed that a decreasing number of symptoms within that cluster were associated with increased functioning as a result of treatment. ${ }^{24}$ In addition, it has been documented among patients suffering from temporomandibular pain that the presence of coexisting conditions was associated with poorer treatment response. ${ }^{25}$ Consistent with these findings, targeting multiple symptoms simultaneously may be a preferable treatment approach for CP patients compared to treating pain in isolation. While these studies have been conducted in the context of $\mathrm{CP}$ and coexisting symptoms or diseases, no research documenting the prevalence and impact of coexisting CP conditions on treatment response among patients attending multidisciplinary pain treatment (MPT) clinics in tertiary care could be found. Given that patients in MPT clinics often receive treatment only for their most bothersome pain condition, it is important to understand whether the presence of other CP conditions impacts on treatment response.

In summary, no studies to our knowledge have examined prevalence and patterns of coexisting CP conditions among patients in tertiary care using precise diagnostic categories. This lack of knowledge contributes to a poor understanding of underlying mechanisms of coexisting CP conditions, and as such, additional research is needed in this field.

The objectives of this study were to determine the prevalence of coexisting CP conditions and identify the most frequent coexisting pain conditions in patients attending MPT clinics, characterize patterns of co-occurrence, examine relationships among patients' biopsychosocial characteristics and number of coexisting $\mathrm{CP}$ conditions, and determine the impact of coexisting $\mathrm{CP}$ conditions on treatment response.

\section{Participants and methods Participants}

Study participants were a subgroup of patients enrolled in the Quebec Pain Registry (QPR; www.quebecpainregistry. com) who provided written consent to use their QPR data for research purposes (91.4\% of patients). The QPR is a large clinical administrative and research database that was implemented to monitor patients' pain conditions over the course of treatment in one of four MPT centers in the province of Quebec (Canada). ${ }^{26}$ Patients enrolled in the QPR were aged 18 years and older, scheduled for a first visit at one of the participating centers, fluent in spoken and written French and/or English, and physically and cognitively able to complete questionnaires. Patients already enrolled in another registry (eg, fibromyalgia registry at one of the participating sites) were not approached. Treatment was not uniform across patients, and was tailored to individual conditions and needs, but could include pharmacological (eg, medications, injections, and blocks) and nonpharmacological (eg, physiotherapy or psychotherapy) approaches. For the purpose of the present study, patients were selected if they were enrolled in the QPR between 2012 and 2014 (time frame for which information on current comorbidities are available), with at 
least one pain diagnosis with pain duration greater than 3 months, and had completed nurse-administered questionnaires prior to the first visit at the pain clinic.

\section{Procedure}

Institutional research-ethics boards of the Centre Hospitalier de l'Université de Montréal, McGill University Health Center, Centre Hospitalier de l'Universite Laval, and the Hôtel-Dieu de Lévis approved the QPR project. QPR patients enrolled between 2012 and 2014 were asked to complete self-report questionnaires (Table 1) before their first visit at the MPT center (baseline patient-administered questionnaire) and 6 months later (6-month patient-administered questionnaire). These questionnaires assessed different pain and pain-related measures, including pain intensity, pain interference, and quality of life. Patient CP diagnoses were provided by the physicians of the pain clinic, who used a standardized grid of diagnoses in which codes were assigned based on pain location, type of condition, and/or suspected etiology. Physicians could enter more than one pain diagnosis, depending on the patient presentation and pain complaints. ${ }^{26}$ Clinical and medical data were also gathered by research nurses using a structured-interview protocol prior to patients' first appointment at the MPT center (baseline nurse-administered questionnaire) and 6 months later (6-month nurse-administered questionnaire). During these interviews, information regarding treatments, medications, and physical/mental comorbidities were collected.

\section{Questionnaires and measures}

\section{Numeric Rating Scale for pain intensity}

The Numeric Rating Scale (NRS) ${ }^{27}$ is a self-administered scale measuring pain intensity ranging from 0 (no pain at all) to 10 (worst possible pain). Psychometric properties of the NRS (in terms of reliability, validity, and sensitivity to change) are good to excellent. ${ }^{27}$ Participants were asked to rate their average pain intensity in the past 7 days.

\section{Brief Pain Inventory 10}

The Brief Pain Inventory (BPI) ${ }^{28,29}$ consists of items assessing pain location (body diagram), medications/treatments, four items measuring pain intensity, and seven items measuring pain interference. In this study, we administered a modified version of the pain-interference items. The original BPI contains seven pain-interference items (activity, mood, mobility, normal work, relationship with others, sleep, and enjoyment in life), to which we added three items (self-care, recreational activities and social activities). ${ }^{30}$ These items assess the extent to which pain impacts on various aspects of daily living in the past 7 days on a scale from 0 (does not interfere) to 10 (completely interferes). A total score composed of the average of each item score is created, with higher average score indicating greater pain interference. Research conducted among CP patients has shown that the BPI has good validity and sensitivity to change. ${ }^{31}$ The BPI has been translated into French using a forward-backward translation method. ${ }^{32}$

\section{Short Form 12 Health Survey version 2}

The Short Form 12 Health Survey version $2^{33}$ assesses physical and mental health-related quality of life using a 12-question survey. Patients are asked for each item to select the statement that best describes their situation. The norm-based summary scores reflecting two different health domains, physical and mental health, based on the general US population, were computed and used in the analyses. Higher scores are indicative of a better quality of life. The survey has good test-retest reliability $(r=0.76-0.89)$ and internal consistency $(a=0.81-0.84)$.

\section{Beck Depression Inventory I}

The Beck Depression Inventory-I (BDI-I) ${ }^{34,35}$ is a 21-item self-report scale measuring levels of depressive symptoms. Each question contains four different statements, and patients are asked to select the statement that best represents the way they are feeling. Each statement is assigned a score from 0 to 3 , and a total score is computed by summing all items. Higher scores are indicative of higher levels of depressive symptomatology. The BDI-I has adequate reliability and validity in various populations. ${ }^{36,37}$

\section{Pain Catastrophizing Scale}

The Pain Catastrophizing Scale ${ }^{38,39}$ is a 13 -item Likert scale measuring ruminative processes, magnification, and helplessness thoughts related to pain experience. For each item, participants rate whether they engage in these thought processes on a scale from 0 (not at all) to 4 (all the time). Higher total scores indicate higher levels of pain catastrophizing. The scale has satisfactory reliability, internal consistency, and validity. ${ }^{38,40,41}$

\section{CP classification}

As part of the QPR, physicians treating enrolled patients were required to use a specific diagnostic grid to provide information on their patients' pain conditions. ${ }^{26}$ This grid had three diagnostic levels: location of pain (eg, thoracic pain, upperlimb pain, generalized pain syndrome), type of disorder 
(eg, postmastectomy pain, fibromyalgia), and suspected etiology (eg, disk disorder, pain following chemotherapy/ radiotherapy).

New guidelines from the ICD $11,{ }^{42}$ currently in its beta version, propose a new classification for CP diseases (see Table 2). Using a hierarchical approach to classify pain conditions (eg, neuropathic pain following surgery classified primarily as postsurgical and posttraumatic pain and only secondarily as neuropathic pain), two of the authors (MGP and MAW) jointly reviewed all possible diagnoses on the QPR diagnostic grid and transferred them into ICD11 CPclassification codes. Each pain-diagnosis code of the QPR was assigned to only one specific category in the ICD11 CP classification (primary pain classification), and secondary classifications were not taken into account.

In addition, the section of the baseline nurse-administered questionnaire devoted to the presence of medical comorbidities was reviewed. In this section, patients were asked to report on any other chronic health conditions they were facing. This section was reviewed to ensure that pain conditions that had not been treated at the MPT center and as such might not have been entered as a pain diagnosis in the QPR by the treating physician were also captured in the analyses. As such, all reported chronic conditions were reviewed and classified into ICD11 codes using the transfer grid established by MGP and MAW.

Pain diagnoses for each patient were reviewed and duplicated diagnoses discarded (eg, postthoracotomy and postsurgical pain in the thoracic region, facet lumbar pain, and disk low-back pain). Two levels of pain classifications were used in the analysis: general pain classes (eg, chronic primary pain) as well as specific pain categories (eg, widespread chronic primary pain, localized chronic primary pain).

\section{Data analyses}

\section{Objective I: prevalence of CP diagnoses and coexisting pain conditions}

Descriptive statistics and $\chi^{2}$ tests were used to examine the prevalence of each pain class and category as well as presence of comorbidities within and between classes and categories.

\section{Objective 2: patterns of co-occurrence}

In order to be included in subsequent analyses, each general pain class (eg, chronic neuropathic pain) required at least 5\% of patients in the sample reporting one or more pain diagnoses within that category and specific pain categories (subgroups of the general pain classes, eg, chronic peripheral neuropathic pain) required at least $1 \%$ of patients in the sample reporting a pain diagnosis within that category. General pain classes and specific pain categories with prevalence inferior to $5 \%$ and $1 \%$, respectively, were excluded from subsequent analyses. A different cutoff was chosen for general pain classes and specific pain categories, since the number of diagnoses differed greatly (seven general pain classes vs 38 specific pain categories). It was not realistic to apply a minimum of 5\% for the specific pain categories, given the sample size and number of specific pain categories.

\section{Factor analysis}

Parallel analysis on the polychoric correlation matrix of the specific pain categories (presence or absence of one or more pain diagnoses within each class) was used to determine the number of factors to retain. Specific pain categories were included in the factor analysis only if they had prevalence greater than $1 \%$ in the sample. Once the number of factors to retain was decided, maximum-likelihood factor analysis on the polychoric correlation matrix with varimax rotation was performed.

\section{Cluster analysis}

Partitioning cluster analysis was used to identify patient clusters that differed in terms of the specific pain categories with a prevalence of at least $1 \%$ in the sample. Cluster analysis was performed on the observations (patients), based on the number of diagnoses within each of the specific pain categories. Solutions ranging from two to 13 clusters were tested and evaluated based on 30 different indicators using the NbClust function in R: Calinski and Harabasz $\mathrm{CH}$ index, Duda index, Pseudot 2 index, C-index, $\gamma$-index, Beale index, Cubic Clustering Criterion, Point biserial index, Gplus index, Davies and Bouldin index, Frey index, Hartigan index, $\tau$-index, Ratkowsky index, Scott index, Marriot index, Ball index, trace of within-cluster pooled covariance matrix index, TraceW index, Friedman index, McClain index, Rubin index, KL index, Silhouette index, Gap index, D index, Dunn index, Hubert statistic, SD validity index, and SDbs validity index. The cluster with the highest number of indicators favoring this solution was retained. Once the number of clusters had been selected, partitioning around medoids was used to obtain the final cluster solution, and descriptive statistics were used to identify the specific pain categories associated with each cluster.

These two data-reduction techniques were selected because they allow for the examination of underlying mechanisms that could explain the observed presence of coexisting conditions. While factor analysis allows for the examination 
of underlying constructs or mechanisms, cluster analysis adopts a subgrouping approach to make sense of the data. ${ }^{43}$ These two approaches were thus used complementarily to examine the patterns of coexisting pain conditions.

\section{Objective 3: relationships among biopsychosocial characteristics and number of coexisting CP conditions}

Negative binomials with log-link generalized linear models were run to test the significance of the association between each of the predictors and number of pain comorbidities (where 0 indicates the patient does not suffer from any pain comorbidity, and thus has only one CP diagnosis). This model was selected given the nominal characteristic of the dependent variable. A total of 11 models were run, one for each individual predictor (age, sex, disability status, education level, pain duration, pain intensity, pain interference, depression, pain catastrophizing, and quality of life [physical and mental] at baseline). Predictors that were statistically significantly associated with number of $\mathrm{CP}$ comorbidities $(P<0.05)$ were entered into a final multivariable generalized linear model. These specific predictors cover three relevant domains to measure in CP patients identified by IMMPACT: ${ }^{44}$ pain, physical functioning, and emotional functioning.

\section{Objective 4: impact of co-occurring CP conditions on treatment responses}

Linear mixed-effect models were used to examine associations among number of pain diagnoses (categorized as 1, 2, or 3+ diagnoses) and pain intensity (model A), pain interference (model B), physical health-related quality of life (model $\mathrm{C})$, and mental health-related quality of life (model D) over the first 6 months (baseline and 6-month follow-up). The number of pain diagnoses was categorized, given the highly skewed distribution of that variable. For each model, time, number of diagnoses, and their interaction were entered as fixed factors, controlling for age and sex. Subjects and time were entered as random effects. Nonsignificant interactions were removed from the model. Effect sizes of associations (Cohen's $d)^{45}$ were also examined, given that significant testing in studies involving large samples like the present one can be misleading, because even small associations can reach statistical significance while clinically viewed as trivial and meaningless. Only differences reaching $d \geq 0.5$ were considered meaningful and clinically important. ${ }^{46-48}$ Linear mixed-effect models were used because they take into account both fixed and random effects and allow for the presence of missing data..$^{43}$
All analyses were conducted in SPSS version 24 and $\mathrm{R}$ version 3.3.0 $0^{49}$ (cluster analysis using the Cluster ${ }^{50}$ and NbClust $^{51}$ packages; factor analysis using the Paramap ${ }^{52}$ and Psych ${ }^{53}$ packages). Given that study participants were included if they had completed the baseline nurse-administered questionnaire, there were no missing data for the prevalence of descriptive comorbidity statistics, factor analysis or cluster analysis. For the generalized linear models and linear mixed models, no data-imputation methods were used.

\section{Results}

\section{Sample characteristics}

A final sample of 3,966 participants was retained for this study (see Figure 1). Descriptive statistics for the overall sample are reported in Table 1. Mean age of participants was 53.39 (SD 14.7) years, and 57.5\% were female, 60.1\% married, and $93.9 \%$ of European descent. Most participants had postsecondary education (55.8\%), but only $21.3 \%$ and $8.7 \%$ were working full-time or part-time, respectively. Approximately a third $(35 \%)$ were on temporary or permanent disability benefits. Participants had a median pain duration of 3 years (mean \pm SD 6.58 \pm 8.62 ) years and an average pain intensity of 6.63/10 (SD 1.98).

\section{Objective I: prevalence of CP diagnoses and co-occurring pain conditions}

Two thirds of participants $(67 \%)$ had only one pain condition, while $23.7 \%, 7.3 \%, 1.5 \%$, and $0.5 \%$ of patients reported two, three, four, and five or more pain conditions, respectively. The median number of pain diagnoses was one (mean \pm SD $1.45 \pm 0.74)$. There was a significant sex difference in number of pain conditions, such that there were more females than males who had three or more pain syndromes $\left(\chi_{3}^{2}=78.75\right.$, $P<0.001)$. Younger participants were more likely to present only one pain condition compared to middle-aged and elderly participants $\left(\chi_{6}^{2}=28.84, P<0.001\right)$. Although statistically significant, all effect sizes of these differences were small ( $\varphi$ - or Cramér's values $<0.3)$. This suggests that these differences were not clinically meaningful. Descriptive statistics on pain and related measures are presented in Table 1 based on number of pain diagnoses.

Prevalence of ICD11 CP-classification general and specific categories are presented in Table 2. The most prevalent pain diagnostic class was chronic musculoskeletal pain (37.9\%), followed by chronic neuropathic pain $(32.2 \%)$, chronic primary pain $(25.4 \%)$, and chronic postsurgical and posttraumatic pain $(20.5 \%)$. The most prevalent specific diagnostic categories were peripheral neuropathic pain 


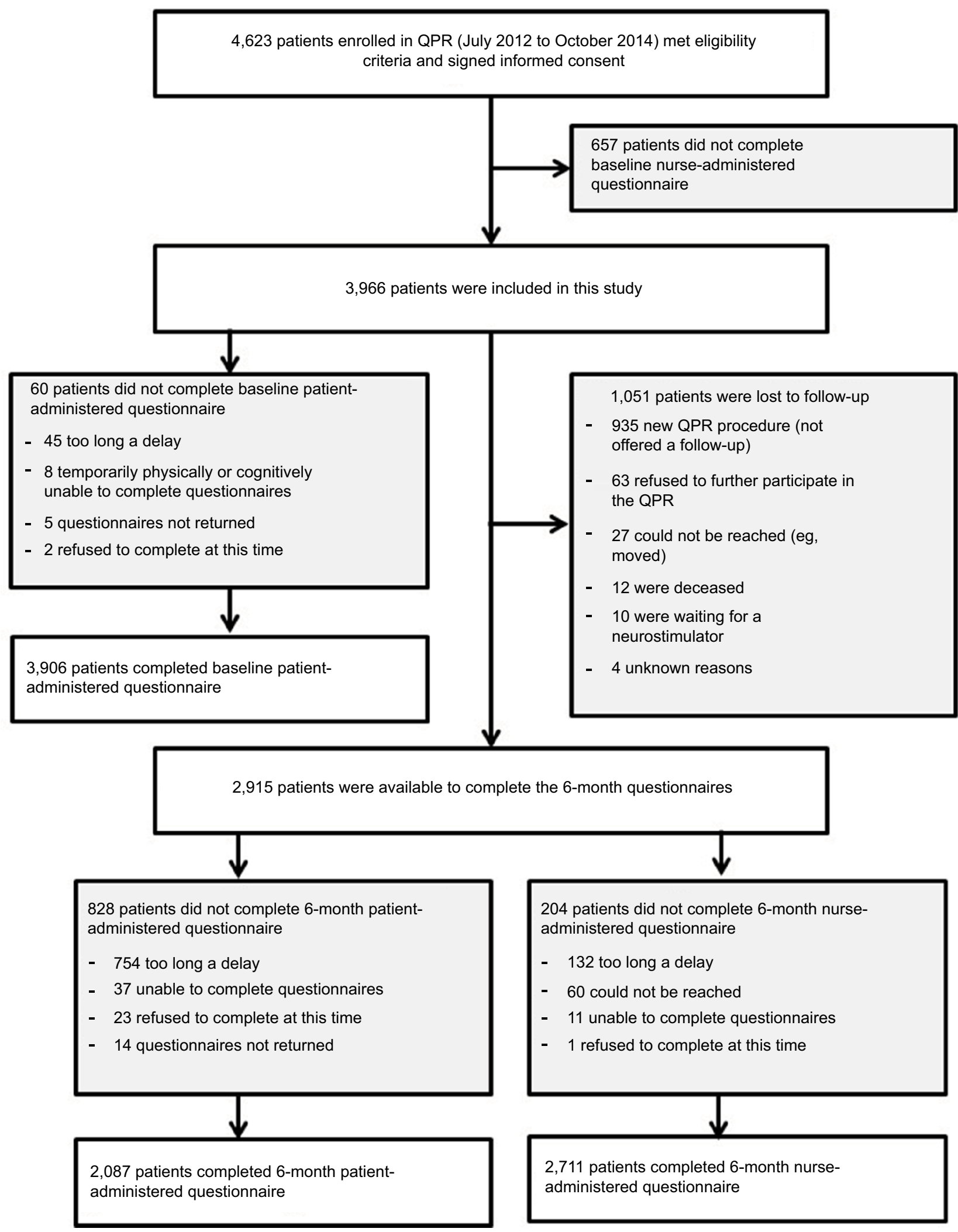

Figure I Study flowchart.

Abbreviation: QPR, Quebec Pain Registry. 
Table I Descriptive statistics for overall sample based on number of pain conditions

\begin{tabular}{|c|c|c|c|c|}
\hline & \multicolumn{3}{|c|}{ Number of pain conditions } & \multirow[t]{2}{*}{ Total } \\
\hline & $\mathbf{I}$ & 2 & $3+$ & \\
\hline Age (years), mean (SD) & $52.87(14.9)$ & $54.13(14.5)$ & $55.37(13.6)$ & $53.4(14.7)$ \\
\hline \multicolumn{5}{|l|}{ Sex, n (\%) } \\
\hline Female & $1,416(53.3)$ & $587(62.5)$ & $276(75.2)$ & $2,279(57.5)$ \\
\hline Male & $\mathrm{I}, 242(46.7)$ & $352(37.5)$ & $91(24.8)$ & $\mathrm{I}, 685(42.5)$ \\
\hline \multicolumn{5}{|l|}{ Baseline, mean (SD) } \\
\hline Pain duration & $5.97(7.9)$ & $7.38(9.5)$ & $8.94(10.8)$ & $6.58(8.6)$ \\
\hline NRS pain intensity & $6.56(2)$ & $6.75(1.9)$ & $6.86(1.8)$ & $6.63(2)$ \\
\hline BPI & $5.56(2.3)$ & $5.73(2.2)$ & $5.74(2.1)$ & $5.62(2.2)$ \\
\hline PCS & $28.68(12.8)$ & $29.88(12.6)$ & $29.8 \mid(\mid 2.6)$ & $29.07(12.8)$ \\
\hline BDI version I & $17.25(10.7)$ & $18.55(10.6)$ & $18.89(10.4)$ & $17.7(10.6)$ \\
\hline SFI 2 version 2 , physical & $29.68(9)$ & $28.17(8.2)$ & $27.91(8.1)$ & $29.17(8.8)$ \\
\hline $\mathrm{SF} 12$ version 2 , mental & $41.42(12)$ & $40.88(12.3)$ & $39.81(11.4)$ & $41.15(12)$ \\
\hline \multicolumn{5}{|l|}{ Six months, mean (SD) } \\
\hline NRS & $5.65(2.4)$ & $5.97(2.3)$ & $6.16(2.1)$ & $5.77(2.4)$ \\
\hline BPI & $4.58(2.6)$ & $4.88(2.4)$ & $4.88(23.3)$ & $4.68(2.5)$ \\
\hline BDI-I & $15.46(10.6)$ & $16.37(10.2)$ & $17.42(10.3)$ & I5.86 (I0.5) \\
\hline SFI 2 version 2 , physical & $32.06(9.8)$ & $30.04(8.8)$ & $29.45(8.8)$ & $31.33(9.5)$ \\
\hline SFI 2 version 2 , mental & $43.34(12.1)$ & $42.86(12)$ & $41.67(11.3)$ & $43.07(12)$ \\
\hline
\end{tabular}

Abbreviations: NRS, Numeric Rating Scale; BPI, Brief Pain Inventory; PCS, Pain Catastrophizing Scale; BDI Beck Depression Inventory; SF, Short Form.

(26.9\%) and chronic musculoskeletal pain from structural osteoarticular changes (21.6\%). Chronic musculoskeletal pain, chronic cancer pain, chronic primary pain, and chronic headache and orofacial pain had the highest average number of pain diagnoses for patients with at least one diagnosis in these categories. Chronic cancer pain (76.5\%), chronic headache and orofacial pain (63.7\%), and chronic visceral pain $(63.6 \%)$ had more than half of patients with a coexisting pain diagnosis from other pain categories.

Descriptive statistics were used to examine the frequency of all pairs of coexisting pain classes (for each possible pair of pain classes, the number of patients with a pain diagnosis in both classes). The most frequent pairs of coexisting pain classes with a minimum of 100 patients reporting those two pain classes are presented in Table $3 . \chi^{2}$ tests showed that the prevalence of patients reporting these pairs of coexisting $\mathrm{CP}$ classes was less than would be expected by chance, based on individual prevalence of each pain class. As such, each of the pain categories was independent of each other and could occur independently from the presence of other coexisting pain conditions. Given that chronic cancer pain and chronic visceral pain were occurring in less than $5 \%$ of patients, these two categories were excluded from further analyses. As such, 43 patients were excluded because they had a pain diagnosis only in these two categories.

\section{Objective 2: patterns of co-occurrence}

A total of 13 specific pain categories were retained for the factor and cluster analyses, as all these categories had prevalence greater than $1 \%$ in the sample (chronic primary pain [1.1 and 1.2], chronic surgical and posttraumatic pain [3.1, 3.2, and 3.x], chronic neuropathic pain [4.1, 4.2, and 4.x], chronic headaches and orofacial pain [5.1 and 5.3] and chronic musculoskeletal pain [7.1, 7.2, and 7.x]).

\section{Factor analysis}

Parallel analysis on 13 of the specific pain categories (with prevalence greater than $1 \%$ in this sample) supported an 11 -factor solution (see Figure 2). All of the factors had only one pain category with high loading $(>0.6)$, and thus no two pain categories had similar patterns of co-occurrence. Eigenvalues and cumulative variance, as well as rotated factor loading, from the maximum-likelihood factor analysis on the polychoric correlation matrix of the 13 specific pain categories are presented in Table 4 and Figure 3, respectively.

\section{Cluster analysis}

Results of the NbClust analysis (series of the 30 indicators evaluating the best cluster solution) of the partitioning cluster analysis testing between two and 13 clusters showed that an 11-cluster solution provided the best fit to the data (see Figure 4). Partitioning around medoids was then used to define the final 11-cluster solution. The median profile for each cluster showed that each variable was associated uniquely with one cluster (ie, no cluster had more than one variable associated with it), with musculoskeletal pain from persistent inflammation and chronic primary headaches not being associated with any one cluster. Similar to results of the factor analysis, 
Table 2 Prevalence of chronic pain conditions based on ICDII (beta version) classification of chronic pain

\begin{tabular}{|c|c|c|c|c|}
\hline & $\begin{array}{l}\text { Patients } \\
\text { with } \geq I C P \\
\text { diagnosis } \\
\text { in category, } \\
n(\%)^{\ddagger} \\
(n=3,966)\end{array}$ & $\begin{array}{l}\text { CP } \\
\text { diagnoses } \\
\text { in category, } \\
n(\%) * \\
(n=5,740)\end{array}$ & $\begin{array}{l}\text { CP diagnoses in } \\
\text { category among } \\
\text { those reporting } \\
\text { at least one } \\
\text { diagnosis in } \\
\text { category, } \\
\text { mean (SD) }\end{array}$ & $\begin{array}{l}\text { Patients within } \\
\text { category with CP } \\
\text { comorbidity } \\
\text { (with other pain } \\
\text { classes), n (\%) }\end{array}$ \\
\hline I. Chronic primary pain & $\mathrm{I}, 008(25.4)$ & & $1.12(0.4)$ & $473(46.9)$ \\
\hline $\begin{array}{l}\text { I.I. Widespread chronic primary pain (including fibromyalgia } \\
\text { syndrome) }\end{array}$ & $352(8.9)$ & $360(6.2)$ & & \\
\hline $\begin{array}{l}\text { 1.2. Localized chronic primary pain (including nonspecific back pain, } \\
\text { chronic pelvic pain) }\end{array}$ & $693(17.4)$ & $750(13.1)$ & & \\
\hline I.x. Other chronic primary pain & $6(0.2)$ & $6(0.1)$ & & \\
\hline I.z. Chronic primary pain not otherwise specified & $14(0.4)$ & $14(0.2)$ & & \\
\hline 2. Chronic cancer pain & $17(0.4)$ & & I.24 (0.6) & $13(76.5)$ \\
\hline 2.I. Chronic pain due to cancer and metastases & $17(0.4)$ & $21(0.5)$ & & \\
\hline \multicolumn{5}{|l|}{$\begin{array}{l}\text { 2.2. Chronic chemotherapy-induced pain (primary parent: chronic } \\
\text { neuropathic pain) }\end{array}$} \\
\hline \multicolumn{5}{|l|}{$\begin{array}{l}\text { 2.3. Chronic pain due to cancer surgery (primary parent: chronic } \\
\text { postsurgical and posttraumatic pain) }\end{array}$} \\
\hline \multicolumn{5}{|l|}{ 2.4. Chronic pain due to radiotherapy } \\
\hline \multicolumn{5}{|l|}{ 2.x. Other chronic pain related to cancer } \\
\hline \multicolumn{5}{|l|}{ 2.z. Chronic cancer pain not otherwise specified } \\
\hline 3. Chronic postsurgical and posttraumatic pain & $812(20.5)$ & & $1.06(0.3)$ & $254(31.3)$ \\
\hline 3.I. Chronic postsurgical pain & $454(11.5)$ & $473(8.2)$ & & \\
\hline 3.2. Chronic posttraumatic pain & $309(7.8)$ & $329(5.7)$ & & \\
\hline 3.x. Other chronic postsurgical and posttraumatic pain & $60(1.5)$ & $60(1)$ & & \\
\hline \multicolumn{5}{|l|}{$\begin{array}{l}\text { 3.z. Chronic postsurgical and posttraumatic pain not otherwise } \\
\text { specified }\end{array}$} \\
\hline 4. Chronic neuropathic pain & $1,279(32.2)$ & & $1.08(0.3)$ & $449(34.9)$ \\
\hline 4.I. Peripheral neuropathic pain & $1,066(26.9)$ & $\mathrm{I}, 139(19.8)$ & & \\
\hline 4.2. Central neuropathic pain & $92(2.3)$ & $93(1.6)$ & & \\
\hline 4.x. Other neuropathic pain & $139(3.6)$ & $|4|(2.5)$ & & \\
\hline 4.z. Neuropathic pain not otherwise specified & $7(0.2)$ & $7(0.1)$ & & \\
\hline 5. Chronic headache and orofacial pain & $377(9.5)$ & & $1.13(0.4)$ & $240(63.7)$ \\
\hline 5.I. Chronic primary headaches & $24 I(6.1)$ & $253(4.4)$ & & \\
\hline 5.2. Chronic secondary headaches & $4(0.1)$ & $4(<0.1)$ & & \\
\hline 5.3. Chronic orofacial pains & $128(3.2)$ & $142(2.4)$ & & \\
\hline 5.z. Headache and orofacial pain not otherwise specified & $28(7.1)$ & $28(0.5)$ & & \\
\hline 6. Chronic visceral pain & $107(2.7)$ & & $1.02(0.1)$ & $68(63.6)$ \\
\hline 6.I. Chronic visceral pain from persistent inflammation & $65(1.6)$ & $66(1.1)$ & & \\
\hline 6.2. Chronic visceral pain from vascular mechanisms & $14(0.4)$ & $14(0.2)$ & & \\
\hline 6.3. Chronic visceral pain from obstruction/distension & $5(0.1)$ & $5(<0.1)$ & & \\
\hline 6.4. Chronic visceral pain from traction/compression & $I(<0.1)$ & I $(<0.1)$ & & \\
\hline \multicolumn{5}{|l|}{ 6.5. Chronic visceral pain from combined mechanisms } \\
\hline \multicolumn{5}{|l|}{ 6.6. Chronic visceral pain referred from other locations } \\
\hline \multicolumn{5}{|l|}{$\begin{array}{l}\text { 6.7. Chronic visceral pain from cancer (primary parent: chronic } \\
\text { cancer pain) }\end{array}$} \\
\hline \multicolumn{5}{|l|}{$\begin{array}{l}\text { 6.8. Functional or unexplained chronic visceral pain (primary parent: } \\
\text { chronic primary pain) }\end{array}$} \\
\hline 6.x. Other chronic visceral pain & $20(0.5)$ & $20(0.3)$ & & \\
\hline 6.z. Chronic visceral pain not otherwise specified & $3(<0.1)$ & $3(<0.1)$ & & \\
\hline
\end{tabular}


Table 2 (Continued)

\begin{tabular}{|c|c|c|c|c|}
\hline & $\begin{array}{l}\text { Patients } \\
\text { with } \geq I \text { CP } \\
\text { diagnosis } \\
\text { in category, } \\
n(\%)^{\ddagger} \\
(n=3,966)\end{array}$ & $\begin{array}{l}\text { CP } \\
\text { diagnoses } \\
\text { in category, } \\
n(\%)^{*} \\
(n=5,740)\end{array}$ & $\begin{array}{l}\text { CP diagnoses in } \\
\text { category among } \\
\text { those reporting } \\
\text { at least one } \\
\text { diagnosis in } \\
\text { category, } \\
\text { mean (SD) }\end{array}$ & $\begin{array}{l}\text { Patients within } \\
\text { category with CP } \\
\text { comorbidity } \\
\text { (with other pain } \\
\text { classes), } n \text { (\%) }\end{array}$ \\
\hline 7. Chronic musculoskeletal pain & $1,502(37.9)$ & & $1.21(0.5)$ & $620(41.3)$ \\
\hline 7.I. Chronic musculoskeletal pain from persistent inflammation & 463 (II.7) & $493(8.6)$ & & \\
\hline $\begin{array}{l}\text { 7.2. Chronic musculoskeletal pain from structural osteoarticular } \\
\text { changes }\end{array}$ & $857(21.6)$ & $941(16.4)$ & & \\
\hline \multicolumn{5}{|l|}{$\begin{array}{l}\text { 7.3. Chronic musculoskeletal pain due to disease of the nervous } \\
\text { system }\end{array}$} \\
\hline \multicolumn{5}{|l|}{$\begin{array}{l}\text { 7.4. Chronic nonspecific musculoskeletal pain (primary parent: } \\
\text { chronic primary pain) }\end{array}$} \\
\hline 7.x. Other chronic musculoskeletal pain syndromes & $353(8.9)$ & $374(6.5)$ & & \\
\hline 7.z. Chronic musculoskeletal pain not otherwise specified & $3(<0.1)$ & $3(<0.1)$ & & \\
\hline
\end{tabular}

Notes: ${ }^{*}$ Numbers within each pain category (eg, I.I, I.2, I.x, I.z) are greater than the total number of diagnoses in the category, because patients with multiple diagnoses within categories (I.2 and I.x) will be counted only once in the overall class; *It is possible for a patient to have multiple diagnoses within a category, eg, nonspecific low-back pain and chronic pelvic pain would both be classified as 1.2 (Localized chronic primary pain), and this patient would have $\mathrm{n}=2$ for this category. Classification of chronic pain for ICDII reproduced with permission from the World Health Organization. ICD-II Beta Draft - Mortality and Morbidity Statistics; 20I7. Available from: https:/licd.who. int/dev I I/l-m/en\#/http\%3a\%2f\%2fid.who.int\%2ficd\%2fentity\%2fl58I 976053 . Accessed November 01, 2017. ${ }^{80}$

Abbreviation: $\mathrm{CP}$, chronic pain.

Table 3 Most frequently co-occurring pairs of coexisting chronic pain classes $(n=3,966)$

\begin{tabular}{|c|c|c|c|c|c|}
\hline & \multirow[t]{2}{*}{$\mathbf{n}$} & \multicolumn{3}{|c|}{ Prevalence/I00 } & \multirow[t]{2}{*}{$\chi^{2}(P$-value $)$} \\
\hline & & Observed* & Expected $^{¥}$ & Observed/expected & \\
\hline CP-MSK & 265 & 6.68 & 9.62 & 0.69 & $77.05(<0.001)$ \\
\hline NP-MSK & 220 & 5.55 & 12.20 & 0.45 & $342.83(<0.001)$ \\
\hline CP-NP & 147 & 3.71 & 8.18 & 0.45 & $193.04(<0.001)$ \\
\hline TR-MSK & 124 & 3.13 & 7.77 & 0.40 & $221.67(<0.001)$ \\
\hline OR-MSK & 113 & 2.85 & 3.6 & 0.79 & $11.05(<0.001)$ \\
\hline
\end{tabular}

Notes: *Number of patients with these two conditions divided by total number of patients in study; ${ }^{*}$ multiplication of individual prevalence of the two comorbid conditions. Abbreviations: NP, chronic neuropathic pain; MSK, chronic musculoskeletal pain; CP, chronic primary pain; TR, chronic postsurgical and posttraumatic pain; OR, chronic headache and orofacial pain.

no two pain categories were found to co-occur. The cluster plot is presented in Figure 5.

\section{Objective 3: relationships among biopsychosocial characteristics and coexisting CP conditions}

Results of the negative binomial with log-link generalized linear models showed that sex, age, pain duration, pain intensity, pain catastrophizing, depression, and physical health-related quality of life were significantly associated at the univariate level with number of coexisting pain conditions, and as such were entered in a final multivariable generalized linear model. The final model was significant (likelihood ratio $\chi^{2}$ $[d f 7]=121.31, P<0.001)$. Table 5 shows parameter estimates for individual variables entered in this model. Results showed that sex, age, pain duration, and physical health-related quality of life were significantly associated with number of coexisting pain conditions.

\section{Objective 4: impact of co-occurring CP conditions on treatment responses}

Only $16 \%$ of the patients had terminated their treatment at the 6-month follow-up. There were no clinically significant differences between treatment status and number of pain conditions $\left(\chi_{2}^{2}=8.33\right.$, Cramér's $\left.\varphi=0.056 ; P=0.016\right)$, and as such this treatment status variable was not included in subsequent analyses.

Results of the linear mixed models $(n=3,830)$ showed no significant interactions between number of pain diagnoses and time in any of the models, and no significant main effect of time $(P>0.05)$. The number of pain diagnoses was significantly associated with pain intensity $(F=3.45$, $P=0.032)$, pain interference $(F=3.29, P=0.038)$, physical health-related quality of life $(F=15.05, P<0.001)$, and mental health-related quality of life $(F=3.48 ; P=0.031)$ after controlling for age and sex. Further examination of the results revealed that patients with only one pain diag- 


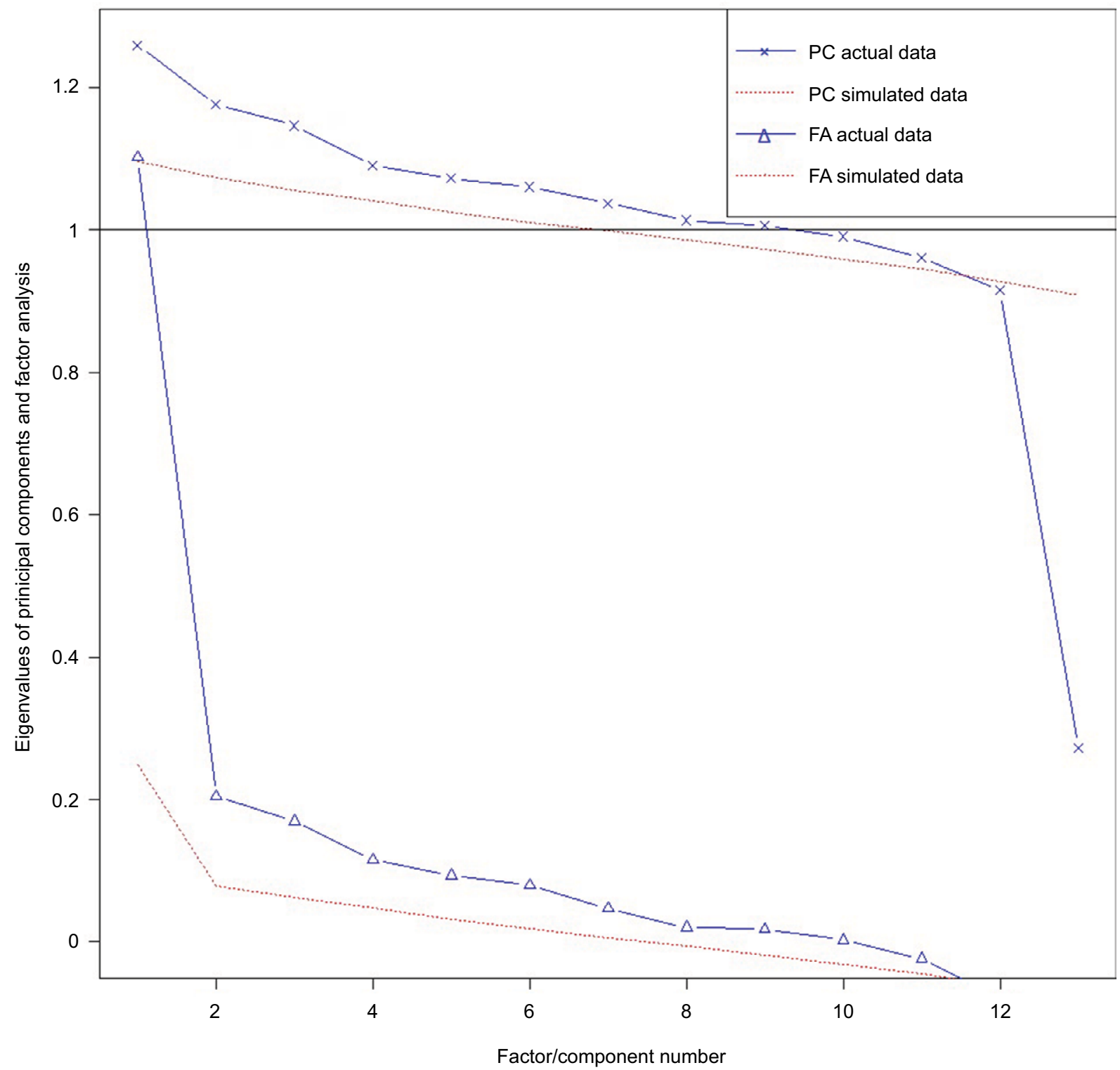

Figure 2 Scree plots with parallel analysis assessing the number of factors to retain. Both approaches suggested an II-factor solution. Abbreviations: PC, principal-component approach; FA, common-factor approach.

Table 4 Eigenvalue and cumulative variance generated using maximum likelihood (ML)-factor analysis on I3 of the specific chronic pain categories with prevalence greater than $1 \%(n=3,903)$

\begin{tabular}{llllllllllll}
\hline & ML4 & MLI & ML3 & ML8 & ML2 & ML6 & ML5 & MLII & ML7 & ML9 & MLI0 \\
\hline Eigenvalue & 1.6 & 1.42 & 1.25 & 1.22 & 1.13 & 1.09 & I & 0.94 & 0.84 & 0.69 & 0.05 \\
Cumulative variance & 0.092 & 0.18 & 0.267 & 0.352 & 0.436 & 0.52 & 0.604 & 0.687 & 0.769 & 0.849 & 0.928 \\
\hline
\end{tabular}

nosis reported significantly lower levels of pain intensity and pain interference compared to patients with two, and three or more pain diagnoses. In addition, patients with one pain diagnosis reported higher levels of physical and mental health-related quality of life compared to the two other groups (see Figure 6). However, the clinical significance of these differences is questionable, considering that all their $d$-values were $<0.5$. 


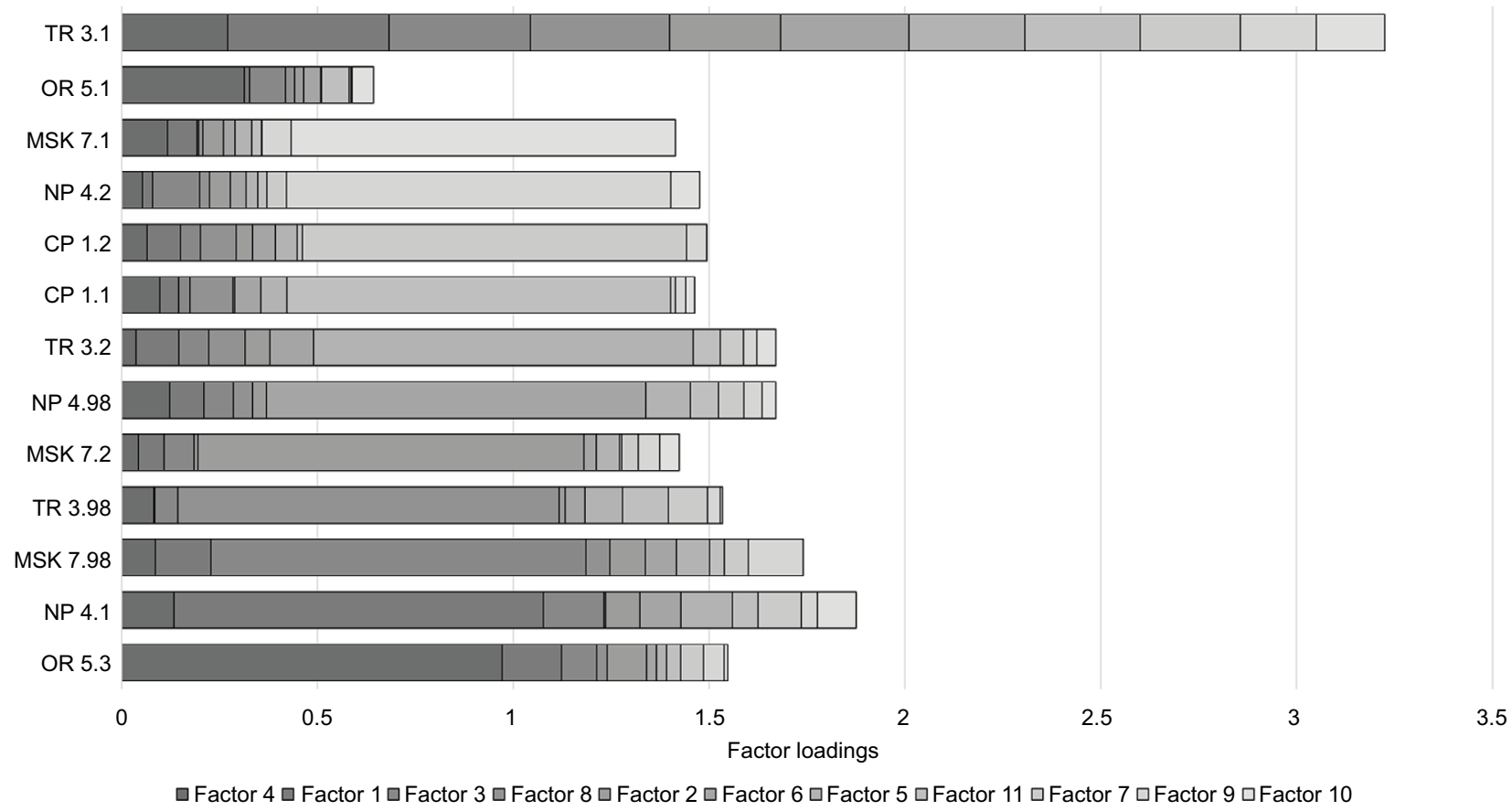

Figure 3 Factor loading for each of the pain diagnoses included in the factor analysis.

Abbreviations: NP, chronic neuropathic pain; MSK, chronic musculoskeletal pain; CP, chronic primary pain; OR, chronic headache and orofacial pain; TR, chronic postsurgical and posttraumatic pain.

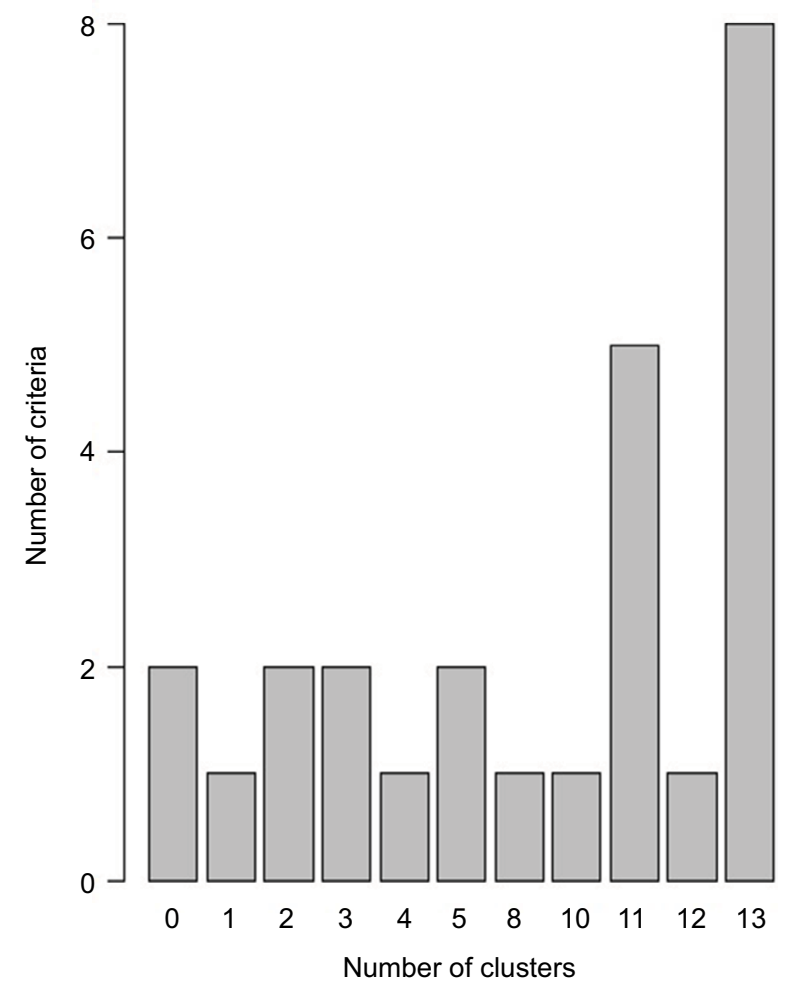

Figure 4 Number of fit indices favoring each of the cluster solutions tested.

\section{Discussion}

Results of this study showed that a third of patients reported coexisting CP conditions, but no specific patterns of co-occurrence of pain comorbidity were identified. The presence of coexisting pain conditions was significantly associated with lower quality of life, longer pain duration, older age, and being female. Contrary to study hypotheses, the presence of coexisting $\mathrm{CP}$ diagnoses did not seem to have a clinically significant impact on treatment responses.

\section{Prevalence of coexisting pain comorbidities}

Results of the present study showed that the mean number of pain diagnoses across classes was 1.45 . These results are similar to those found in some population studies. For example, results from the World Mental Health Survey showed that $41 \%$ of participants with $\mathrm{CP}$ reported having more than one painful condition. ${ }^{12}$ Another large-scale study using claim databases found a mean number of diagnoses of 1.39-2.65 depending on the primary pain condition..$^{54}$ Other studies focusing on specific pain populations also found similar rates of coexistence. Among patients with chronic low-back pain, up to $40 \%$ of these patients also reported other pain conditions, such as neck and middle/upper-back pain, musculoskeletal pain, or neuropathic pain. ${ }^{11}$ Two-thirds of patients with chronic spinal pain reported the presence of other CP conditions, and this was associated with role disability. ${ }^{16}$

When examining the occurrence of coexisting pain conditions, it is important to differentiate between coexisting pain diagnoses and multiple pain sites. Examining the number of 


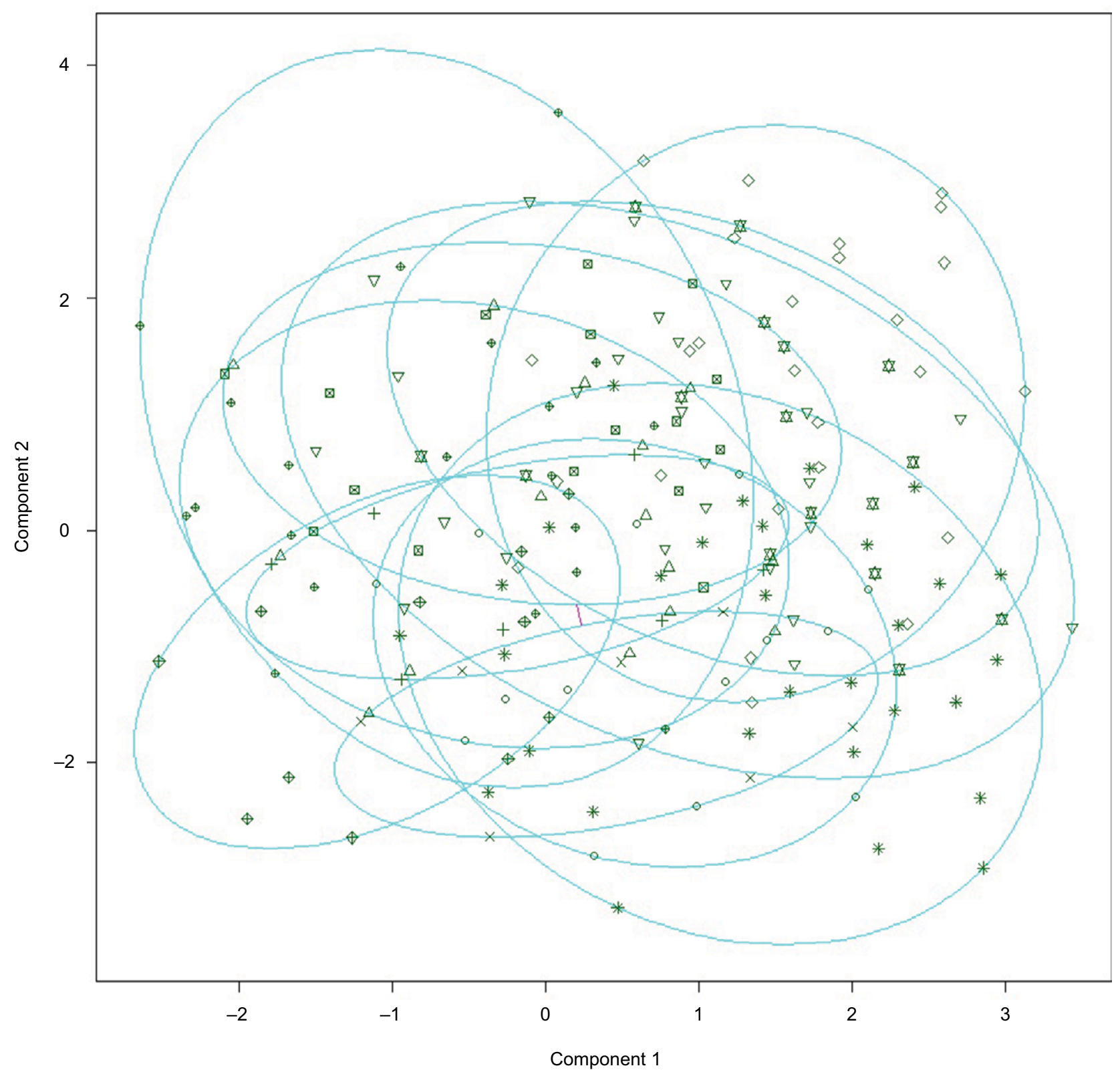

Figure 5 Bivariate cluster plot of the 13-cluster solution.

Note: These two components explain $18.73 \%$ of the point variability.

Table 5 Negative binomial generalized linear model examining associations between number of comorbid chronic pain conditions and sociodemographic and biopsychosocial characteristics at baseline $(n=3,906)$

\begin{tabular}{|c|c|c|c|c|c|c|}
\hline & \multirow[t]{2}{*}{ Wald $\chi^{2}$} & \multirow[t]{2}{*}{$P$-value } & \multirow[t]{2}{*}{$\boldsymbol{\beta}$} & \multirow[t]{2}{*}{ SE } & \multicolumn{2}{|l|}{$95 \% \mathrm{Cl}$} \\
\hline & & & & & Lower bound & Upper bound \\
\hline Intercept & 878.27 & $<0.001$ & -1.19 & 0.05 & -1.29 & -1.09 \\
\hline Sex - female & 57.84 & $<0.001$ & 0.48 & 0.06 & 0.36 & 0.61 \\
\hline \multicolumn{7}{|l|}{ Sex - male } \\
\hline Age & 7.03 & 0.008 & 0.01 & 0.002 & 0.002 & 0.01 \\
\hline Pain duration & 25.24 & $<0.001$ & 0.02 & 0.003 & 0.01 & 0.02 \\
\hline Average NRS pain intensity & 0.22 & 0.640 & 0.01 & 0.02 & -0.03 & 0.04 \\
\hline PCS & 0.001 & 0.989 & 0.001 & 0.003 & -0.01 & 0.01 \\
\hline BDI-I & 2 & 0.158 & 0.01 & 0.004 & -0.002 & 0.01 \\
\hline SFI 2 version 2 , physical & 9.39 & 0.002 & -0.01 & 0.004 & -0.02 & -0.004 \\
\hline
\end{tabular}

Abbreviations: NRS, Numeric Rating Scale; PCS, Pain Catastrophizing Scale; BDI Beck Depression Inventory; SF, Short Form. 

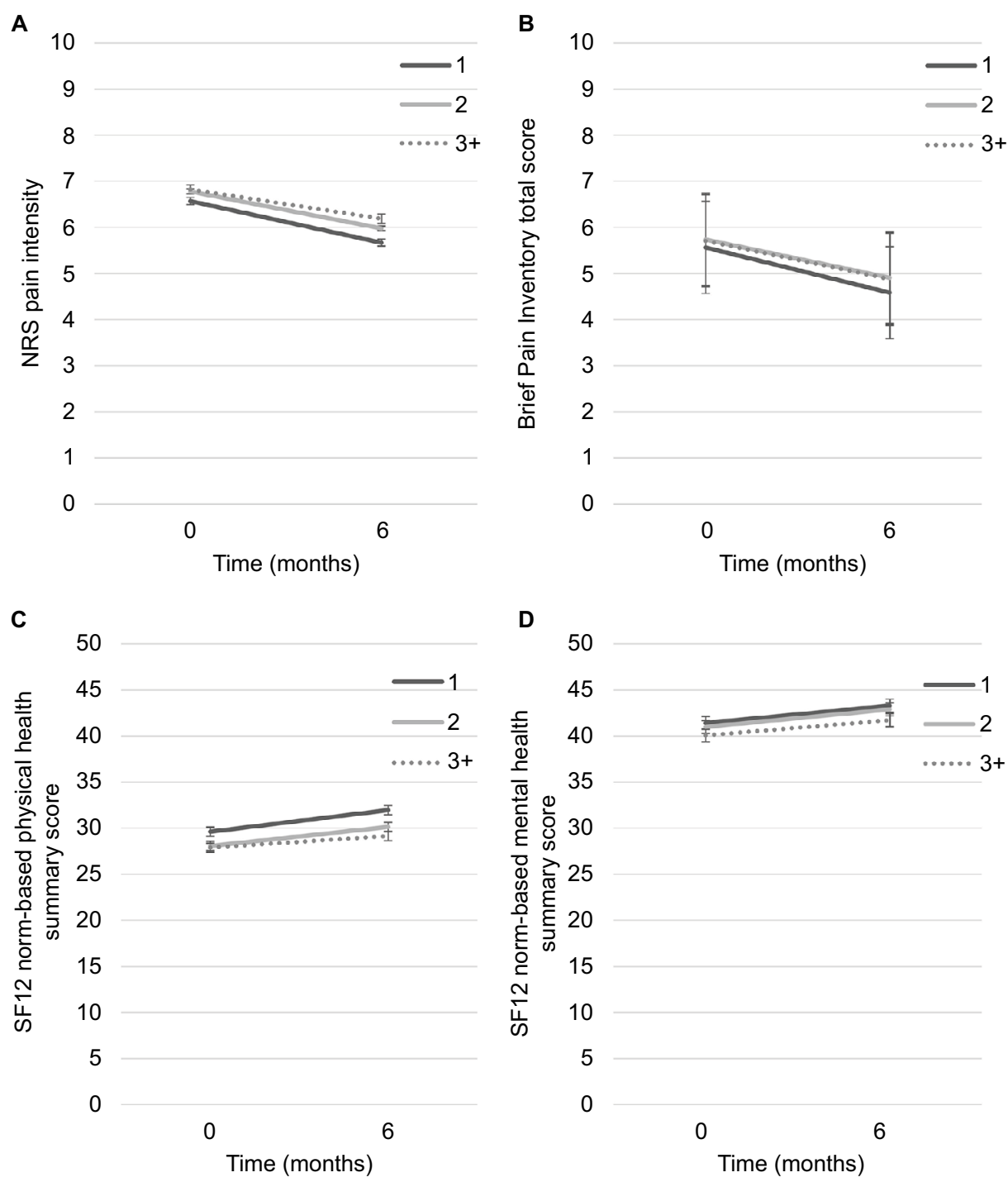

Figure 6 Results of the linear mixed models.

Notes: Statistically significant associations between number of pain diagnoses and pain intensity (A), pain interference (B), physical health-related quality of life (C), and mental health-related quality of life (D).

Abbreviations: NRS, Numeric Rating Scale; SF, Short Form.

pain sites is not the same as measuring the co-occurrence of different pain conditions, since the latter counts multiple pain sites as only one pain condition if it originates from the same chronic disease entity.

Some studies focusing on coexisting pain conditions among specific CP cohorts offer some hypotheses that could explain the observed rates of comorbidity. A study of patients with temporomandibular conditions showed that compared to matched controls, these patients had higher odds of reporting coexisting pain conditions; they also experienced lower pressure-pain thresholds and higher levels of somatic symptoms. ${ }^{55}$ These results suggest that generalized alterations in pain processing might underlie the observed coexistence of pain conditions. Animal models may also offer new insights into the comorbidity of coexisting pain conditions, such as stress-induced hypersensitivity. ${ }^{56}$

Our results suggest that coexisting pain conditions across pain classes are not present as often as would be expected by chance. They also suggest that when pain conditions coexist, they seem independent of the type of CP (nociceptive vs neuropathic). In fact, among the top five coexisting pain conditions, three pairs involved a mixture of neuropathic and nociceptive types of pain (eg, neuropathic pain and musculoskeletal pain, chronic primary pain and neuropathic pain, and postsurgical/posttraumatic pain and musculoskeletal pain). Interestingly, these results suggest that the presence of coexisting pain conditions is not dependent on a unique type of pain. It is possible that predisposing and vulnerability 
factors make one person more likely to develop CP, whether it is neuropathic or nociceptive pain. An increasing amount of research is being published supporting the role of genes and gene expression in pain pathways. ${ }^{57-59}$ In addition, other studies suggest that the presence of one pain type (eg, neuropathic pain) might lead to the development of nociceptive pain through inflammatory mechanisms (neurogenic inflammation). ${ }^{60}$ There is also an emerging body of literature supporting the concept of central sensitization syndromes that could explain the comorbidity between such syndromes as fibromyalgia, irritable bowel syndrome, chronic low-back pain, and other CP conditions in terms of shared pathophysiological mechanisms. ${ }^{61}$

A growing body of literature has defined chronic overlapping pain conditions ${ }^{62}$ to represent a cluster of CP conditions that are frequently seen together and share some degrees of abnormalities of neurologic, endocrine, and immune systems; these include fibromyalgia, temporomandibular disorders, chronic fatigue syndrome, irritable bowel syndrome, interstitial cystitis, endometriosis, chronic tension-type and migraine headache, and chronic low-back pain. Many of these disorders are classified as chronic primary pain in the ICD11 classification. Results from this study showed that patients with at least one diagnosis in this category had an average of 1.13 conditions classified as chronic primary pain. This was not the highest rate of coexistence: chronic cancer pain and chronic musculoskeletal pain had higher rates of co-occurrence. Therefore, while chronic overlapping pain conditions might represent a specific entity of comorbidities, results showed that it is also important to investigate co-occurrence of CP conditions beyond this specific cluster of conditions.

In terms of pain management, one could expect that co-occurrence of CP syndromes would bring an additional difficulty, since treatments that would alleviate neuropathic pain are often different from those that would relieve nociceptive pain (eg, first-line pharmacological treatment of neuropathic pain would include anticonvulsants and antidepressants, whereas opioids would be preferable for nociceptive pain). ${ }^{63,64}$

\section{Patterns of coexisting pain comorbidities}

The purpose of the factor analysis and cluster analysis was to examine whether a specific pattern of coexistence (ie, identifying some CP categories that are frequently present simultaneously within patients) was identifiable. Results did not allow the identification of specific patterns of coexisting CP conditions. Both factor and cluster analyses used to examine patterns of coexisting pain comorbidities identified nearly as many groups of pain categories $(n=11)$ as the number of pain categories examined $(n=13)$. The consistency observed across these two different statistical approaches strengthens the results within this study. Results are nonetheless in contrast with those observed from other studies. For example, two studies showed that having pain at one body site puts someone at higher risk of pain at another site, especially those located physically closer to one another (eg, back and neck) ${ }^{10}$ or bilaterally. ${ }^{65}$ These studies, however, examined the comorbidity of pain sites in the general population. It is possible that underlying patterns of co-occurrence exist for different CP locations and that these patterns do not reflect a common pattern of underlying origin or etiology of the pain experience.

Little research has been conducted on patterns of coexisting pain conditions using data-reduction approaches. Some studies have examined such patterns in the context of physical and mental comorbidities. For example, among patients 17 years of age, four distinct comorbidity clusters were found (low probability of low-back pain or other diagnosis; high probability of low-back pain and neck/shoulder pain; low-back pain conditions and mood/anxiety conditions; and low-back pain and behavioral and attention conditions). ${ }^{66}$ These different clusters also had different impact in terms of quality of life and CP burden.

Taken together, results from this study suggest that while one person might have a specific vulnerability to develop coexisting pain conditions, the occurrence of this comorbidity does not seem to follow a specific type (neuropathic/nociceptive) or pattern. These results could partially be explained by the pain-classification method used and the overlap among certain categories. The ICD11 beta CP classification hierarchically classifies pain conditions as a function of primary and secondary diagnoses. ${ }^{42}$ For example, neuropathic pain from a surgical procedure will be classified as chronic postsurgical pain as the primary parent and peripheral neuropathic pain as a secondary class. As such, it is possible that the potential overlap between specific categories made it difficult to identify patterns of multiple pain classes. In addition, it is also possible that the mere fact of experiencing one pain condition puts someone at risk of developing another pain condition, regardless of pain location. Consistent with findings from the Orofacial Pain Prospective Evaluation and Risk Assessment study, ${ }^{67}$ Diatchenko et al ${ }^{68}$ suggested that $\mathrm{CP}$ (regardless of peripheral location) was similarly regulated and that common factors (environmental, psychosocial, genetic) predisposed an individual to develop multiple CP conditions. 
Therefore, the lack of an identifiable pattern to $\mathrm{CP}$ coexistence in this study is in line with this conceptualization of underlying common regulating systems rendering a patient with $\mathrm{CP}$ more at risk of developing another $\mathrm{CP}$ condition, irrespective of its peripheral location.

\section{Predictors of number of pain conditions}

Results from this study showed that sex, age, pain duration, and physical health-related quality of life might be associated with number of pain comorbidities. The latter represented the overall number of independent pain diagnoses and could be within or across specific pain categories. Consistent with other research, results suggest that different risk factors exist for having one versus coexisting pain conditions. ${ }^{65,69}$ Being female and older were factors associated with the presence of a higher number of pain conditions. These results are consistent with other studies in which sex, age, and health-related quality of life significantly predicted reports of multiple pain sites. ${ }^{65,70-72}$

In the present study, longer pain duration was associated with increased number of pain conditions. Literature on this topic is controversial. Some studies have shown that the number of pain sites does not increase over time, such that patients reporting fewer pain sites at one time point are also very likely to report few pain sites at a later time. ${ }^{70}$ In contrast, the presence of additional pain conditions among patients with temporomandibular conditions was associated with longer pain duration. ${ }^{73}$ In addition, other research has found that the presence of musculoskeletal pain in one body region increases the chance of later reporting pain in other body regions. ${ }^{74}$ Other studies have also demonstrated similar associations. $^{73,75}$

\section{Associations with treatment outcomes}

Results of the present study did not find a significant interaction between time and number of pain classes in predicting pain and related outcomes over the first 6 months of MPT. This suggests that the presence of coexisting pain conditions would not impact on treatment responses. The number of pain classes was statistically significantly associated with pain intensity, interference, and quality of life after controlling for age and sex. However, given the large sample size of this study, the examination of effect sizes (clinical significance) is often a better indicator of the degree of association between variables than statistical significance, such as $P$-values. ${ }^{46}$ Results of this study showed that none of the associations between number of pain classes and pain intensity, interference, or quality of life was of moderate or large effect size (Cohen's $d<0.5$ ). Even though cutoff values on such indicators as Cohen's $d$ are typically used to guide interpretation of results, it suggests that the clinical significance of the results obtained in this study are questionable. As such, one can conclude that patients with pain diagnosis(es) in only one pain class do not different from those who have pain diagnoses across multiple pain classes in terms of pain intensity, interference, or quality of life.

These results are generally in contrast with the literature. In fact, many studies have documented an association between coexisting pain conditions/sites, pain interference, occupation, and psychosocial factors. ${ }^{69,70,74,76,77}$ Past research has also suggested that the presence of pain comorbidities among specific pain diagnoses might signify the presence of more complex pain conditions that would be associated with greater disease burden and require specialized treatments. ${ }^{61,78}$ Conclusions from this study might differ from the existing literature, because they take into account the clinical significance of the results given the large sample size and are based on number of pain conditions and not the number of pain sites. It is also possible that other features of the pain experience, such as prior pain episodes, previous treatment response, and pain duration, have a greater impact on treatment response than co-occurring pain conditions. In addition, since the majority of patients seen at an MPT clinic are more complex and have a relatively minimal response to treatment, ${ }^{79}$ the overall small treatment response in the current study sample might have made it difficult to identify changes in treatment response based on number of pain diagnoses.

\section{Strengths and limitations}

This study was based on a large sample of patients attending MPT at tertiary-care clinics in Quebec, Canada. Pain diagnoses were based on the ICD11 beta version of CP classification, which is the most current and optimal pain classification, which will ensure comparability of results with future studies. ${ }^{42}$ Nonetheless, this study also has limitations. First, in order to capture most accurately the global pain experience of patients, self-reported pain diagnoses were included as part of the pain conditions, in addition to doctor-generated pain diagnoses. This was important to count the number of pain conditions accurately, since most MPT clinics focus only on treating the most disruptive pain conditions a patient presents with and as such number of pain conditions might be underreported. Second, the study was based on a specific pain population accessing specialized pain treatments in the tertiary sector of care, and as such, results cannot be generalized to patients treated in other sectors (primary care) or to the general population. In 
addition, treatments received were individualized and varied across patients and between clinics. Nonetheless, all patients were treated for their primary pain condition, and the analyses examined whether having additional pain conditions interfered with this treatment response. Last, only primary pain classifications were retained for the purpose of the analyses, such that each pain condition was attributed to a unique pain class. This was done to facilitate data analysis, but also introduce some representation bias, since there is overlap naturally occurring among the pain classes.

\section{Agenda for future research and conclusions}

This study has demonstrated that the presence of coexisting pain conditions is prevalent in CP patients and is associated with demographic and pain characteristics and psychosocial factors. The occurrence of coexisting pain conditions does not seem to follow specific patterns, and occurs across pain classes. The impact of coexisting pain diagnoses on pain outcomes has not been clearly established. This study was conducted at the tertiary-care level, where patients typically present with more complex, treatment-resistant CP conditions. While patterns of coexisting pain conditions do not appear to impact treatment response at this level, it will be important for future research to examine whether this remains true among patients at earlier stages of the CP experience. It will also be important to replicate these findings using other pain populations and comparing different methodologies for pain classification.

\section{Acknowledgments}

The authors thank all the QPR nurses and assistants for their dedicated work during data collection at the multidisciplinary pain-treatment clinics of the CHUM, MUHC, CHUL, and HDL. Thanks are also due to the medical directors of these pain clinics, to all physicians working in each participating site, and to the patients who gave consent for their QPR data to be used for research purposes.

This study was funded by the Quebec Pain Research Network (QPRN), which is itself funded by governmental grants from the agencies Fonds de la Recherche du QuébecSanté (FRQS) and Quebec Ministry of Health. The QPRN is also supported by private funding from Pfizer Canada and Astra-Zeneca, whose contributions were channeled through the FRQS via an official financial partnership.

\section{Disclosure}

$\mathrm{MC}$ received funding from the Research Center of the CHUM to carry out this study. MGP is currently a recipient of a postdoctoral research award from the Canadian Institutes of Health Research. MAW was a research scholar of the FRQS. The authors report no other conflicts of interest in this work.

\section{References}

1. Merskey H, Bogduk N. Classification of Chronic Pain: Descriptions of Chronic Pain Syndromes and Definitions of Pain Terms. 2nd ed. Washington: International Association for the Study of Pain; 1994.

2. [No authors listed]. Classification of chronic pain: descriptions of chronic pain syndromes and definitions of pain terms. Pain Suppl. 1986;3:S1-S226.

3. Schopflocher D, Taenzer P, Jovey R. The prevalence of chronic pain in Canada. Pain Res Manag. 2011;16(6):445-450.

4. Moulin DE, Clark AJ, Speechley M, Morley-Forster PK. Chronic pain in Canada: prevalence, treatment, impact and the role of opioid analgesia. Pain Res Manag. 2002;7(4):179-184.

5. van Hecke O, Torrance N, Smith BH. Chronic pain epidemiology and its clinical relevance. Br J Anaesth. 2013;111(1):13-18.

6. Croft P. Disease-related pain: an introduction. In: Croft P, Blyth FM, van der Windt D, editors. Chronic Pain Epidemiology: From Aetiology to Public Health. Oxford: Oxford University Press; 2010: 203-208.

7. Siddall PJ, Cousins MJ. Persistent pain as a disease entity: implications for clinical management. Anesth Analg. 2004;99(2):510-520.

8. Kirsh KL. Differentiating and managing common psychiatric comorbidities seen in chronic pain patients. J Pain Palliat Care Pharmacother. 2010;24(1):39-47.

9. Dominick CH, Blyth FM, Nicholas MK. Unpacking the burden: understanding the relationships between chronic pain and comorbidity in the general population. Pain. 2012;153(2):293-304.

10. Ligthart L, Visscher CM, van Houtem CM, et al. Comorbidity among multiple pain symptoms and anxious depression in a Dutch population sample. J Pain. 2014;15(9):945-955.

11. Gore M, Sadosky A, Stacey BR, Tai KS, Leslie D. The burden of chronic low back pain: clinical comorbidities, treatment patterns, and health care costs in usual care settings. Spine (Phila Pa 1976). 2012;37(11):E668-E677.

12. Gureje O, Von Korff M, Kola L, et al. The relation between multiple pains and mental disorders: results from the World Mental Health surveys. Pain. 2008;135(1-2):82-91.

13. Riedl A, Schmidtmann M, Stengel A, et al. Somatic comorbidities of irritable bowel syndrome: a systematic analysis. J Psychosom Res. 2008;64(6):573-582.

14. Croft P, Dunn KM, Von Korff M. Chronic pain syndromes: you can't have one without another. Pain. 2007;131(3):237-238.

15. Picavet HS, Schouten JS. Musculoskeletal pain in the Netherlands: prevalences, consequences and risk groups, the $\mathrm{DMC}_{3}$-study. Pain. 2003;102(1-2):167-178.

16. Von Korff M, Crane P, Lane M, et al. Chronic spinal pain and physicalmental comorbidity in the United States: results from the national comorbidity survey replication. Pain. 2005;113(3):331-339.

17. van Hecke O, Torrance N, Smith BH. Chronic pain epidemiology: where do lifestyle factors fit in? Br J Pain. 2013;7(4):209-217.

18. Fillingim RB. Sex, gender, and pain: women and men really are different. Curr Rev Pain. 2000;4(1):24-30.

19. Fillingim RB, King CD, Ribeiro-Dasilva MC, Rahim-Williams B, Riley JL, 3rd. Sex, gender, and pain: a review of recent clinical and experimental findings. J Pain. 2009;10(5):447-485.

20. Unruh AM. Gender variations in clinical pain experience. Pain. 1996;65(2-3):123-167.

21. Kehlet H, Jensen TS, Woolf CJ. Persistent postsurgical pain: risk factors and prevention. Lancet. 2006;367(9522):1618-1625.

22. Katz J. One man's risk factor is another man's outcome: difference in risk factor profiles for chronic postsurgical pain maintenance vs transition. Pain. 2012;153(3):505-506. 
23. Branco JC, Bannwarth B, Failde I, et al. Prevalence of fibromyalgia: a survey in five European countries. Semin Arthritis Rheum. 2010;39(6):448-453.

24. Davis LL, Kroenke K, Monahan P, Kean J, Stump TE. The SPADE symptom cluster in primary care patients with chronic pain. Clin $J$ Pain. 2016;32(5):388-393.

25. Raphael KG, Marbach JJ. Widespread pain and the effectiveness of oral splints in myofascial face pain. J Am Dent Assoc. 2001;132(3): 305-316

26. Choiniere M, Ware MA, Page MG, et al. Development and implementation of a registry of patients attending multidisciplinary pain treatment clinics: the Quebec Pain Registry. Pain Res Manag. 2017;2017: 8123812 .

27. Jensen MP, Karoly P. Self-report scales and procedures for assessing pain in adults. In: Turk DC, Melzack R, editors. Handbook of Pain Assessment. 2nd ed. New York: Guilford Press; 2001:15-34.

28. Cleeland CS, Ryan KM. Pain assessment: global use of the Brief Pain Inventory. Ann Acad Med Singapore. 1994;23(2):129-138.

29. Cleeland CS, Nakamura Y, Mendoza TR, Edwards KR, Douglas J, Serlin RC. Dimensions of the impact of cancer pain in a four country sample: new information from multidimensional scaling. Pain. 1996;67(2-3):267-273.

30. Tyler EJ, Jensen MP, Engel JM, Schwartz L. The reliability and validity of pain interference measures in persons with cerebral palsy. Arch Phys Med Rehabil. 2002;83(2):236-239.

31. Tan G, Jensen MP, Thornby JI, Shanti BF. Validation of the Brief Pain Inventory for chronic nonmalignant pain. J Pain. 2004;5(2): 133-137.

32. Larue F, Carlier AM, Brasseur L, Colleau SM, Cleeland CS. Assessing the prevalence and severity of cancer pain in France: the French Brief Pain Inventory. Poster presented at: American Pain Society 10th Annual Scientific Meeting; November 7-10, 1991; New Orleans, LA.

33. Ware J Jr, Kosinski M, Keller SD. A 12-item short-form health survey: construction of scales and preliminary tests of reliability and validity. Med Care. 1996;34(3):220-233.

34. Beck AT, Ward CH, Mendelson M, Mock J, Erbaugh J. An inventory for measuring depression. Arch Gen Psychiatry. 1961;4:561-571.

35. Bourque P, Beaudette D. Etude psychométrique du questionnaire de dépression de Beck auprès d'un échantillon d'étudiants universitaires francophones. Can J Behav Sci. 1982;14:211-221.

36. Beck A, Steer RA, Garbin GM. Psychometric properties of the Beck Depression Inventory: twenty-five years of evaluation. Clin Psychol Rev. 1988;8(1):77-100.

37. Beck AT, Steer RA. Internal consistencies of the original and revised Beck Depression Inventory. J Clin Psychol. 1984;40(6):1365-1367.

38. Sullivan MJ, Bishop SR, Pivik J. The Pain Catastrophizing Scale: development and validation. Psycho Assess. 1995;7(4):524-532.

39. French DJ, Noël M, Vigneau F, French JA, Cyr CP, Evans RT. A Frenchlanguage, French-Canadian adaptation of the Pain Catastrophizing Scale. Can J Behav Sci. 2005;37:181-192.

40. Sullivan MJL, Lynch ME, Clark MR. Dimensions of catastrophic thinking associated with pain experience and disability in patients with neuropathic pain conditions. Pain. 2005;113(3):310-315.

41. Sullivan MJ, Thorn B, Haythornthwaite JA, et al. Theoretical perspectives on the relation between catastrophizing and pain. Clin J Pain. 2001;17(1):52-64

42. Treede RD, Rief W, Barke A, et al. A classification of chronic pain for ICD-11. Pain. 2015;156(6):1003-1007.

43. Tabachnick BG, Fidell LS. Using Multivariate Statistics. 5th ed. London: Pearson; 2006.

44. Turk DC, Dworkin RH, Allen RR, et al. Core outcome domains for chronic pain clinical trials: IMMPACT recommendations. Pain. 2003;106(3):337-345.

45. Cohen J. Statistical Power Analysis for Behavioral Sciences. Mahwah (NJ): Lawrence Erlbaum Associates; 1988.

46. Wilkinson L. Statistical methods in psychology journals: guidelines and explanations. Am Psychol. 1999;54(8):594-604.
47. Kline RB. Beyond Significance Testing. Washington: American Psychological Association; 2004.

48. Gliklich R, Dreyer N. Registries for Evaluating Patient Outcomes: A User's Guide. 3rd ed. Washington: US Department of Health and Human Services; 2014.

49. R Project. R: a language and environment for statistical computing. Available from: http://www.R-project.org. Accessed November 14, 2017.

50. Maechler M, Rousseeuw M, Struyf A, Hubert M, Hornik K. Cluster: cluster analysis basics and extensions. 2017. Available from: https:// cran.r-project.org/web/packages/cluster/index.html. Accessed April $17,2017$.

51. Charrad M, Ghazzali N, Boiteau V, Niknafs A. NbClust: an R package for determining the relevant number of clusters in a data set. J Stat Softw. 2014;61(6):1-36.

52. O’Connor BP. Paramap: R package version 1.1. 2016. Available from:https://people.ok.ubc.ca/brioconn/nfactors/paramap.pdf. Accessed January 10, 2017.

53. Revelle W. Psych: procedures for personality and psychological research. 2016. Available from: http://personality-project.org/r/psychmanual.pdf. Accessed November 14, 2017.

54. Davis JA, Robinson RL, Le TK, Xie J. Incidence and impact of pain conditions and comorbid illnesses. J Pain Res. 2011;4:331-345.

55. Chen H, Slade G, Lim PF, Miller V, Maixner W, Diatchenko L. Relationship between temporomandibular disorders, widespread palpation tenderness, and multiple pain conditions: a case-control study. J Pain. 2012;13(10):1016-1027.

56. Traub RJ, Cao DY, Karpowicz J, et al. A clinically relevant animal model of temporomandibular disorder and irritable bowel syndrome comorbidity. J Pain. 2014;15(9):956-966

57. Foulkes T, Wood JN. Pain genes. PLoS Genet. 2008;4(7):e1000086.

58. He X, Fan L, Wu Z, He J, Cheng B. Gene expression profiles reveal key pathways and genes associated with neuropathic pain in patients with spinal cord injury. Mol Med Rep. 2017;15(4):2120-2128.

59. Descalzi G, Mitsi V, Purushothaman I, et al. Neuropathic pain promotes adaptive changes in gene expression in brain networks involved in stress and depression. Sci Signal. 2017;10(471):aaj1549.

60. Chiu IM, von Hehn CA, Woolf CJ. Neurogenic inflammation and the peripheral nervous system in host defense and immunopathology. Nat Neurosci. 2012;15(8):1063-1067.

61. Kindler LL, Bennett RM, Jones KD. Central sensitivity syndromes: mounting pathophysiologic evidence to link fibromyalgia with other common chronic pain disorders. Pain Manag Nurs. 2011;12(1):15-24.

62. Veasley C, Clare D, Clauw D, et al. Impact of chronic overlapping pain conditions on public health and the urgent need for safe and effective treatment: 2015 analysis and policy recommendations. 2015. Available from: http://www.chronicpainresearch.org/public/CPRA_ WhitePaper_2015-FINAL-Digital.pdf. Accessed November 14, 2017.

63. Moulin D, Boulanger A, Clark AJ, et al. Pharmacological management of chronic neuropathic pain: revised consensus statement from the Canadian Pain Society. Pain Res Manag. 2014;19(6):328-335.

64. Dworkin RH, O'Connor AB, Audette J, et al. Recommendations for the pharmacological management of neuropathic pain: an overview and literature update. Mayo Clinic Proc. 2010;85(3 Suppl):S3-S14.

65. Coggon D, Ntani G, Palmer KT, et al. Patterns of multisite pain and associations with risk factors. Pain. 2013;154(9):1769-1777.

66. Beales DJ, Smith AJ, O'Sullivan PB, Straker LM. Low back pain and comorbidity clusters at 17 years of age: a cross-sectional examination of health-related quality of life and specific low back pain impacts. J Adolesc Health. 2012;50(5):509-516.

67. Maixner W, Diatchenko L, Dubner R, et al. Orofacial pain prospective evaluation and risk assessment study: the OPPERA study. J Pain. 2011;12(11 Suppl):T4-T11.e1-e2.

68. Diatchenko L, Nackley AG, Slade GD, Fillingim RB, Maixner W. Idiopathic pain disorders: pathways of vulnerability. Pain. 2006;123(3): 226-230.

69. Kamaleri Y, Natvig B, Ihlebaek CM, Bruusgaard D. Localized or widespread musculoskeletal pain: does it matter? Pain. 2008;138(1):41-46. 
70. Kamaleri Y, Natvig B, Ihlebaek CM, Benth JS, Bruusgaard D. Change in the number of musculoskeletal pain sites: a 14-year prospective study. Pain. 2009;141(1-2):25-30.

71. Lacey RJ, Belcher J, Rathod T, Wilkie R, Thomas E, McBeth J. Pain at multiple body sites and health-related quality of life in older adults: results from the North Staffordshire Osteoarthritis Project. Rheumatology (Oxford). 2014;53(11):2071-2079.

72. Kamaleri Y, Natvig B, Ihlebaek CM, Benth JS, Bruusgaard D. Number of pain sites is associated with demographic, lifestyle, and health-related factors in the general population. Eur J Pain. 2008;12(6):742-748.

73. Dahan H, Shir Y, Velly A, Allison P. Specific and number of comorbidities are associated with increased levels of temporomandibular pain intensity and duration. $J$ Headache Pain. 2015;16:528.

74. Andersen LL, Clausen T, Carneiro IG, Holtermann A. Spreading of chronic pain between body regions: prospective cohort study among health care workers. Eur J Pain. 2012;16(10):1437-1443.

75. Grimby-Ekman A, Gerdle B, Björk J, Larsson B. Comorbidities, intensity, frequency and duration of pain, daily functioning and health care seeking in local, regional, and widespread pain: a descriptive populationbased survey (SwePain). BMC Musculoskelet Disord. 2015;16:165.
76. Miranda H, Kaila-Kangas L, Heliovaara M, et al. Musculoskeletal pain at multiple sites and its effects on work ability in a general working population. Occup Environ Med. 2010;67(7):449-455.

77. Landro NI, Fors EA, Vapenstad LL, Holthe O, Stiles TC, Borchgrevink PC. The extent of neurocognitive dysfunction in a multidisciplinary pain centre population: is there a relation between reported and tested neuropsychological functioning? Pain. 2013;154(7):972-977.

78. Lorduy KM, Liegey-Dougall A, Haggard R, Sanders CN, Gatchel RJ. The prevalence of comorbid symptoms of central sensitization syndrome among three different groups of temporomandibular disorder patients. Pain Pract. 2013;13(8):604-613.

79. Pagé G, Escobar EM, Ware MA, Choinière M. Predicting treatment outcomes of pain patients attending tertiary multidisciplinary pain treatment centers: a pain trajectory approach. Can J Pain. 2017;1(1): $61-74$.

80. World Health Organization. ICD-11 Beta Draft - Mortality and Morbidity Statistics; 2017. Available from: https://icd.who.int/dev11/1-m/en\#/ http\%3a\%2f\%2fid.who.int $\% 2$ ficd $\% 2$ fentity $\% 2$ f1581976053. Accessed November 01, 2017.
Journal of Pain Research

\section{Publish your work in this journal}

The Journal of Pain Research is an international, peer reviewed, open access, online journal that welcomes laboratory and clinical findings in the fields of pain research and the prevention and management of pain. Original research, reviews, symposium reports, hypothesis formation and commentaries are all considered for publication.

\section{Dovepress}

The manuscript management system is completely online and includes a very quick and fair peer-review system, which is all easy to use. Visit http://www.dovepress.com/testimonials.php to read real quotes from published authors. 